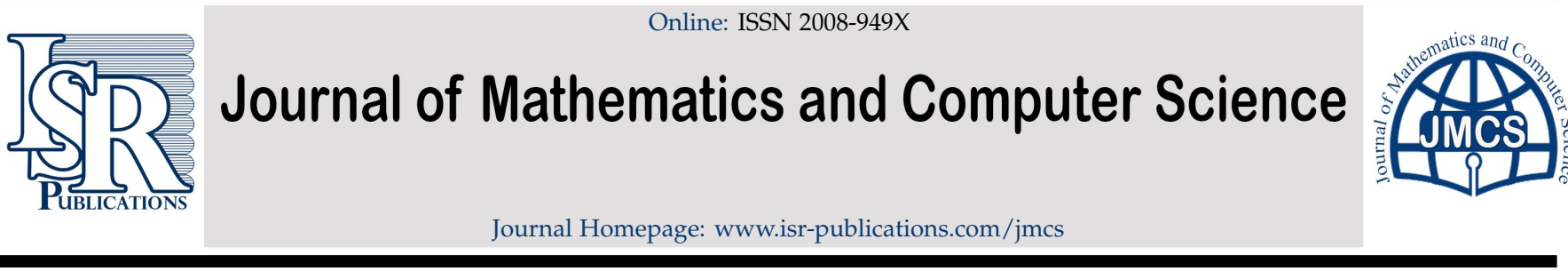

\title{
A note on modified Hermite matrix polynomials
}

\author{
Virender Singh ${ }^{\mathrm{a}}$, Mumtaz Ahmad Khan ${ }^{\mathrm{b}}$, Abdul Hakim Khan ${ }^{\mathrm{b}}$, Kottakkaran Sooppy Nisar ${ }^{\mathrm{c}, *}$ \\ ${ }^{a}$ Department of Applied Mathematics, Galgotias college of Engineering and Technology, Greater Noida, Uttar Pradesh-201306, India. \\ ${ }^{b}$ Department of Applied Mathematics, Faculty of Engineering and Technology, Aligarh Muslim University, Aligarh-202002, India. \\ ${ }^{c}$ Department of Mathematics, College of Arts and Sciences, Wadi Aldawaser, Prince Sattam bin Abdulaziz University, 11991, Saudi \\ Arabia.
}

\begin{abstract}
The main aim of this paper is to investigate the modified Hermite matrix polynomials ${ }_{\mathrm{M}} \mathscr{H}_{\mathrm{n}}\left(\zeta_{1}, \lambda ; \mathscr{A}\right)$ by finding some important results such as generating functions, recurrence relations, Rodrigues formula, orthogonality conditions, expansion formula, integrals, fractional integrals, fractional derivatives and some other properties.
\end{abstract}

Keywords: Gamma matrix function, hypergeometric matrix function, three term matrix recurrence relation, modified Hermite matrix differential equation, modified Hermite matrix polynomials, orthogonal matrix polynomials.

2020 MSC: 33C05, 33C45, 33C90.

(C)2021 All rights reserved.

\section{Introduction and preliminaries}

An opening explanation to the class of Hermite matrix polynomials $\mathscr{H}_{\mathrm{n}}\left(\zeta_{1} ; \mathscr{A}\right)$ and some basic properties have been considered in [2, 4-7, 10, 11, 13, 22, 23]. A comprehensive view on Hermite matrix polynomials are given in [12, 26-29]. Jódar and co-authors established the classical families of Hermite matrix polynomials $\mathscr{H}_{\mathrm{n}}\left(\zeta_{1} ; \mathscr{A}\right)$ defined by

$$
\exp \left(\zeta_{1} \mathfrak{z} \sqrt{2 \mathscr{A}}-\mathfrak{z}^{2} \mathrm{I}\right)=\sum_{\mathrm{n} \geqslant 0} \frac{\mathscr{H}_{\mathrm{n}}\left(\zeta_{1} ; \mathscr{A}\right) \mathfrak{z}^{\mathrm{n}}}{\mathrm{n} !}
$$

where

$$
\mathscr{H}_{\mathrm{n}}\left(\zeta_{1} ; \mathscr{A}\right)=\sum_{\mathrm{k}=0}^{\left[\frac{\mathrm{n}}{2}\right]} \frac{(-1)^{\mathrm{k}} \mathrm{n} !\left(\zeta_{1} \sqrt{2 \mathscr{A}}\right)^{\mathrm{n}-2 \mathrm{k}}}{\mathrm{k} !(\mathrm{n}-2 \mathrm{k}) !}, \quad \mathrm{n} \geqslant 0,
$$

where $\mathscr{A}$ is a +ve stable matrix in the complex space $\mathbb{C}^{\mathrm{N} \times \mathrm{N}}$ of all square matrices of common order $\mathrm{N}$ which appear as a finite series solution of second order matrix differential equation $y^{\prime \prime}-\zeta_{1} \mathscr{A} y^{\prime}+n \mathscr{A} y=$

\footnotetext{
*Corresponding author

Email addresses: virenderamu2015@gmail.com (Virender Singh), mumtaz_ahmad_khan_2008@yahoo.com (Mumtaz Ahmad Khan), ahkhan . amu@gmail.com (Abdul Hakim Khan), n. sooppy@psau.edu .sa (Kottakkaran Sooppy Nisar)
}

doi: $10.22436 /$ jmcs.022.04.03

Received: 2020-05-27 Revised: 2020-06-30 Accepted: 2020-07-23 
0 , for a matrix $\mathscr{A}$ in $\mathbb{C}^{\mathrm{N} \times \mathrm{N}}$ whose eigenvalues are all in the right open half plane. It has been given by Defez and Jódar $[1,4]$ the matrices $\mathscr{A}(k, n)$ and $\mathscr{B}(k, n)$ in $\mathbb{C}^{N \times N}$ where $n \geqslant 0, k \geqslant 0$, the following relations are satisfied,

$$
\sum_{n \geqslant 0} \sum_{k \geqslant 0} \mathscr{A}(k, n)=\sum_{n \geqslant 0} \sum_{k=0}^{[n / 2]} \mathscr{A}(k, n-2 k),
$$

and

$$
\sum_{n=0}^{\infty} \sum_{k=0}^{\infty} \mathscr{B}(k, n)=\sum_{n=0}^{\infty} \sum_{k=0}^{n} \mathscr{B}(k, n-k) .
$$

Similarly, we can write

$$
\begin{aligned}
\sum_{n=0}^{\infty} \sum_{k=0}^{[n / 2]} \mathscr{A}(k, n) & =\sum_{n=0}^{\infty} \sum_{k=0}^{\infty} \mathscr{A}(k, n+2 k), \\
\sum_{n=0}^{\infty} \sum_{k=0}^{n} \mathscr{B}(k, n) & =\sum_{n=0}^{\infty} \sum_{k=0}^{[n / 2]} \mathscr{B}(k, n-k), \\
\sum_{n=0}^{\infty} \sum_{k=0}^{n} \mathscr{A}(k, n) & =\sum_{n=0}^{\infty} \sum_{k=0}^{\infty} \mathscr{A}(k, n+k) .
\end{aligned}
$$

Also, if $\mathscr{A}+n I$ is invertible for every integer $n \geqslant 0$, then form [9]. The matrix version of the Pochhammer symbol is

$$
\begin{aligned}
& (\mathscr{A})_{\mathrm{n}}=\Gamma(\mathscr{A}+\mathrm{nI}) \Gamma(\mathscr{A})^{-1}, \\
& (\mathscr{A})_{\mathrm{n}}= \begin{cases}\mathrm{I}, & \text { if } \mathrm{n}=0 \\
\mathscr{A}(\mathscr{A}+\mathrm{I})(\mathscr{A}+2 \mathrm{I}) \cdots(\mathscr{A}+(\mathrm{n}-1) \mathrm{I}), & \text { if } \mathrm{n}=1,2, \cdots .\end{cases}
\end{aligned}
$$

From (1.2), it is easy to calculate that

$$
(\mathscr{A})_{\mathrm{n}-\mathrm{k}}=(-1)^{\mathrm{k}}(\mathscr{A})_{\mathrm{n}}(\mathrm{I}-\mathscr{A}-\mathrm{nI})_{\mathrm{k}}{ }^{-1} ; \quad 0 \leqslant \mathrm{k} \leqslant \mathrm{n} .
$$

From [25, pp-58], one can obtain

$$
\frac{(-1)^{k}}{(n-k) !} I=\frac{(-n)_{k}}{n !} I=\frac{(-n I)_{k}}{n !} ; \quad 0 \leqslant k \leqslant n,
$$

if $\mathscr{A}+\mathrm{nI} \mathscr{B}+\mathrm{nI}$, and $\mathscr{A}+\mathscr{B}+\mathrm{nI}$ all are invertible

$$
\beta(\mathscr{A}, \mathscr{B})=\frac{\Gamma(\mathscr{A}) \Gamma(\mathscr{B})}{\Gamma(\mathscr{A}+\mathscr{B})} ; \quad 0 \leqslant \mathrm{k} \leqslant \mathrm{n},
$$

where $\beta(\mathscr{A}, \mathscr{B})$ denotes beta matrix function for the pair $\mathscr{A}, \mathscr{B}$. The hypergeometric matrix function $\mathrm{F}(\mathscr{A}, \mathscr{B} ; \mathscr{C} ; z)$ has been given in the form $[9,14]$ for matrix $\mathscr{A}, \mathscr{B}$, and $\mathscr{C}$ in $\mathbb{C}^{\mathrm{N} \times \mathrm{N}}$ such that $\mathscr{C}+\mathrm{nI}$ is invertible for all integer $n \geqslant 0$,

$$
\mathrm{F}(\mathscr{A}, \mathscr{B} ; \mathscr{C} ; z)=\sum_{\mathrm{n} \geqslant 0} \frac{(\mathscr{A})_{\mathrm{n}}(\mathscr{B})_{\mathrm{n}}}{(\mathscr{C})_{\mathrm{n}} \mathrm{n} !} z^{\mathrm{n}},
$$

it converges for $|z|<1$, for any matrix $\mathscr{A}$ in $\mathbb{C}^{\mathrm{N} \times \mathrm{N}}$ we will make use of the following relation due to [9],

$$
\left(1-\zeta_{1}\right)^{-\mathscr{A}}=\sum_{n \geqslant 0} \frac{(\mathscr{A})_{n} \zeta_{1}^{n}}{n !} ; \quad\left|\zeta_{1}\right|<1 .
$$

For more details about various type of polynomials and its properties, one can be referred to $[15,16$, $18-20,24,31]$. 
The paper is organized as follows. In Section 2, we collect the definition of the modified Hermite

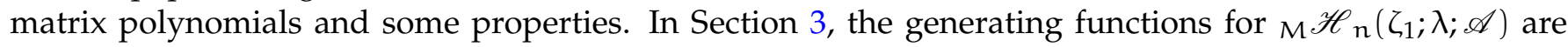
introduced. In Sections 4, 5, and 6 we find recurrence relations, hypergeometric form and Rodrigues formula for the modified Hermite matrix polynomials. In Sections 7, 8, and 9, we obtain orthogonality, expansion of polynomials and some integral representation for $M_{M} \mathscr{H}_{n}\left(\zeta_{1} ; \lambda ; \mathscr{A}\right)$. Finally in Section 10 , we present fractional integrals and derivatives for modified Hermite matrix polynomials.

\section{The Definition of ${ }_{M} \mathscr{H}_{\mathbf{n}}\left(\zeta_{1} ; \lambda ; \mathscr{A}\right)$}

The modified Hermite matrix polynomials $\mathrm{M}_{\mathscr{H}_{\mathrm{n}}}\left(\zeta_{1} ; \lambda ; \mathscr{A}\right)$ can be obtained by means of generating relation

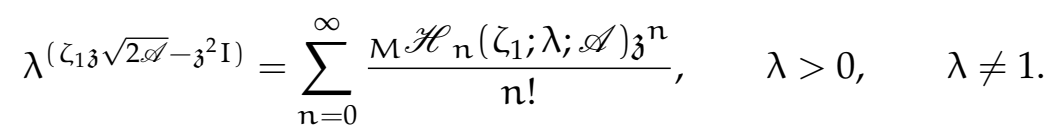

Using equation (2.1), we have

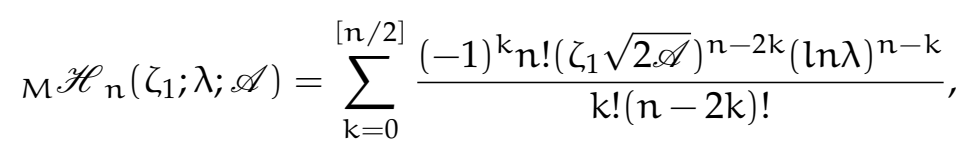

for $\lambda=e$, it reduces to Hermite Matrix polynomials $\mathscr{H}_{\mathrm{n}}\left(\zeta_{1}, \mathscr{A}\right)$ which have been considered by Jódar's and Company [7]. In equation (2.1), if we replace $\zeta_{1}$ by $-\zeta_{1}$ and $\mathfrak{z}$ by $-\mathfrak{z}$ left hand side of the equation (2.1) does not change, so

$$
\mathrm{M} \mathscr{H}_{\mathrm{n}}\left(\zeta_{1} ; \lambda ; \mathscr{A}\right)=(-1)^{\mathrm{n}}{ }_{\mathrm{M}} \mathscr{H}_{\mathrm{n}}\left(\zeta_{1} ; \lambda ; \mathscr{A}\right) \text {. }
$$

It shows that ${ }_{M} \mathscr{H}_{\mathrm{n}}\left(\zeta_{1} ; \lambda ; \mathscr{A}\right)$ is an odd function of $\zeta_{1}$ for odd $\mathrm{n}$, an even function of $\zeta_{1}$ for even $\mathrm{n}$. Also

$$
\mathrm{M} \mathscr{H}_{2 \mathrm{n}}(0 ; \lambda ; \mathscr{A})=(-1)^{\mathrm{n}} 2^{2 \mathrm{n}}\left(\frac{1}{2}\right)_{\mathrm{n}}(\ln \lambda)^{\mathrm{n}}, \quad \mathrm{M}^{\mathscr{H}_{2 \mathrm{n}+1}}(0 ; \lambda ; \mathscr{A})=0,
$$

and

$$
\mathrm{M}^{\mathscr{H}_{2 \mathrm{n}+1}^{\prime}}(0 ; \lambda ; \mathscr{A})=(-1)^{\mathrm{n}} 2^{2 \mathrm{n}}\left(\frac{3}{2}\right)_{\mathrm{n}} \sqrt{2 \mathscr{A}}(\ln \lambda)^{\mathrm{n}+1}, \quad \mathrm{M}^{\mathscr{H}_{2 \mathrm{n}}^{\prime}}(0 ; \lambda ; \mathscr{A})=0
$$

The first few modified Hermite matrix polynomials are

$$
\begin{aligned}
& \mathrm{M}^{\mathscr{H}_{0}}\left(\zeta_{1} ; \lambda ; \mathscr{A}\right)=\mathrm{I} \text {, } \\
& \mathrm{M}^{\mathscr{H}_{1}}\left(\zeta_{1} ; \lambda ; \mathscr{A}\right)=\zeta_{1} \sqrt{2 \mathscr{A}}(\ln \lambda) \\
& \mathrm{M} \mathscr{H}_{2}\left(\zeta_{1} ; \lambda ; \mathscr{A}\right)=\left(\zeta_{1} \sqrt{2 \mathscr{A}}\right)^{2}(\ln \lambda)^{2}-2(\ln \lambda) \mathrm{I} \text {, } \\
& \mathrm{M} \mathscr{H}_{3}\left(\zeta_{1} ; \lambda ; \mathscr{A}\right)=\left(\zeta_{1} \sqrt{2 \mathscr{A}}\right)^{3}(\ln \lambda)^{3}-6\left(\zeta_{1} \sqrt{2 \mathscr{A}}\right)(\ln \lambda)^{2}, \\
& \mathrm{M}^{\mathscr{H}_{4}}\left(\zeta_{1} ; \lambda ; \mathscr{A}\right)=\left(\zeta_{1} \sqrt{2 \mathscr{A}}\right)^{4}(\ln \lambda)^{4}-12\left(\zeta_{1} \sqrt{2 \mathscr{A}}\right)^{2}(\ln \lambda)^{3}+12 \ln \lambda \mathrm{I} \text {, } \\
& \mathrm{M}^{\mathscr{H}_{5}}\left(\zeta_{1} ; \lambda ; \mathscr{A}\right)=\left(\zeta_{1} \sqrt{2 \mathscr{A}}\right)^{5}(\ln \lambda)^{5}-20\left(\zeta_{1} \sqrt{2 \mathscr{A}}\right)^{3}(\ln \lambda)^{4}+60\left(\zeta_{1} \sqrt{2 \mathscr{A}}\right)(\ln \lambda)^{3} \text {, } \\
& \mathrm{M}^{\mathscr{H}_{6}}\left(\zeta_{1} ; \lambda ; \mathscr{A}\right)=\left(\zeta_{1} \sqrt{2 \mathscr{A}}\right)^{6}(\ln \lambda)^{6}-30\left(\zeta_{1} \sqrt{2 \mathscr{A}}\right)^{4}(\ln \lambda)^{5}+180\left(\zeta_{1} \sqrt{2 \mathscr{A}}\right)^{2}(\ln \lambda)^{4}-120(\ln \lambda)^{3} \mathrm{I} \text {. }
\end{aligned}
$$

\section{Generating function for $\mathrm{M}_{\mathfrak{H}} \mathscr{H}_{\mathrm{n}}\left(\zeta_{1} ; \lambda ; \mathscr{A}\right)$}

In this section, we give the following generating functions.

\section{Brafman type generating function}

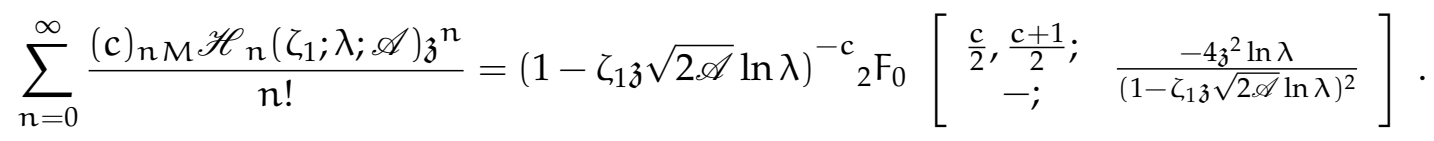




\section{Bilinear generating function}

$$
\sum_{n=0}^{\infty} \frac{M \mathscr{H}_{\mathrm{n}}\left(\zeta_{1} ; \lambda ; \mathscr{A}\right)_{M} \mathscr{H}_{\mathrm{n}}\left(\zeta_{2} ; \lambda ; \mathscr{A}\right) \mathfrak{z}^{\mathrm{n}}}{n !}=\left(1-4 \mathfrak{z}^{2} \ln \lambda\right)^{-\frac{1}{2}} \lambda^{\frac{2 \mathscr{A} \mathfrak{z} \ln \lambda\left(\zeta_{1} \zeta_{2}-\zeta_{1}^{2} \ln \lambda-\zeta_{2}^{2} \mathfrak{z}\right)}{\left(1-4_{\mathfrak{z}} \ln \lambda\right)}}
$$

\section{Other generating functions:}

Some other generating functions are as follows:

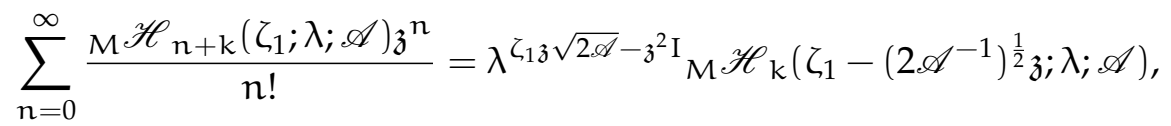

$$
\begin{aligned}
& \sum_{n=0}^{\infty} \frac{{ }_{2} \mathrm{~F}_{0}\left[-\mathrm{n}, \mathrm{c} ;-; \zeta_{2}\right]_{\mathrm{M}} \mathscr{H}_{\mathrm{n}}\left(\zeta_{1} ; \lambda ; \mathscr{A}\right) \mathfrak{z}^{\mathrm{n}}}{\mathrm{n} !}=\lambda^{\zeta_{1} \mathfrak{z} \sqrt{2 \mathscr{A}}-\mathfrak{z}^{2} \mathrm{I}}\left(1+\zeta_{2} \mathfrak{z} \sqrt{2 \mathscr{A}}\left(\zeta_{1}-{\sqrt{2 \mathscr{A}^{-1}}}^{-\mathrm{c}}\right.\right.
\end{aligned}
$$

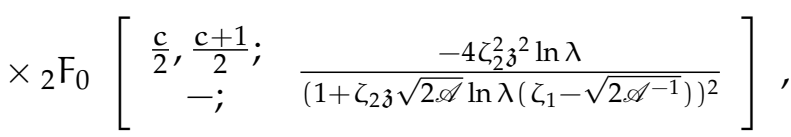

$$
\begin{aligned}
& \sum_{n=0}^{\infty} \frac{(-1)^{\mathrm{n}}{ }_{M} \mathscr{H}_{2 n}\left(\zeta_{1} ; \lambda ; \mathscr{A}\right) \mathfrak{z}^{\mathrm{n}}}{(2 \mathrm{n}) !}=\lambda^{\mathfrak{z}} \cos \left(\zeta_{1} \ln \lambda \sqrt{2 \mathscr{A} \mathfrak{z}}\right), \\
& \sum_{n=0}^{\infty} \frac{(-1)^{n} M \mathscr{H}_{2 n+1}\left(\zeta_{1} ; \lambda ; \mathscr{A}\right) \mathfrak{z}^{n}}{(2 \mathrm{n}+1) !}=\mathfrak{z}^{-\frac{1}{2}} \lambda^{\mathfrak{z}} \sin \left(\zeta_{1} \ln \lambda \sqrt{2 \mathscr{A} \mathfrak{z}}\right), \\
& \sum_{n=0}^{\infty} \frac{M \mathscr{H}_{2 n}\left(\zeta_{1} ; \lambda ; \mathscr{A}\right) \mathfrak{z}^{2 n}}{(2 n) !}=\lambda^{-\mathfrak{z}^{2}} \cosh \left(\zeta_{1} \mathfrak{z} \ln \lambda \sqrt{2 \mathscr{A}}\right), \\
& \sum_{n=0}^{\infty} \frac{M \mathscr{H}_{2 n+1}\left(\zeta_{1} ; \lambda ; \mathscr{A}\right) \mathfrak{z}^{2 n+1}}{(2 n+1) !}=\lambda^{-\mathfrak{z}^{2}} \sinh \left(\zeta_{1 \mathfrak{z}} \ln \lambda \sqrt{2 \mathscr{A}}\right), \\
& \sum_{n=0}^{\infty} \frac{(c)_{n M} \mathscr{H}_{2 n}\left(\zeta_{1} ; \lambda ; \mathscr{A}\right) \mathfrak{z}^{2 n}}{(2 n) !}=\left(1+\mathfrak{z}^{2} \ln \lambda\right)^{-c}{ }_{1} F_{1}\left[c ; \frac{1}{2} ; \frac{\zeta_{1}^{2} \mathfrak{z}^{2}(\ln \lambda)^{2}(\sqrt{2 \mathscr{A}})^{2}}{4\left(1+\mathfrak{z}^{2} \ln \lambda\right)}\right] \text {. }
\end{aligned}
$$

Proof of (3.1).

$$
\begin{aligned}
& \sum_{n=0}^{\infty} \frac{(\mathrm{c})_{n M} \mathscr{H}_{n}\left(\zeta_{1} ; \lambda ; \mathscr{A}\right) \mathfrak{z}^{n}}{n !}=\sum_{n=0}^{\infty} \sum_{k=0}^{[n / 2]} \frac{(-1)^{k}(c){ }_{n}\left(\zeta_{1} \sqrt{2 \mathscr{A}}\right)^{n-2 k}(\ln \lambda)^{n-k} \mathfrak{z}^{n}}{k !(n-2 k) !} \\
& =\sum_{n=0}^{\infty} \sum_{k=0}^{\infty} \frac{(-1)^{k}(c) n_{n+2 k}\left(\zeta_{1} \sqrt{2 \mathscr{A}}\right)^{n}(\ln \lambda)^{n+k} \mathfrak{z}^{n+2 k}}{k ! n !} \\
& =\sum_{k=0}^{\infty} \sum_{n=0}^{\infty} \frac{(c+2 k)_{n}\left(\zeta_{1} \sqrt{2 \mathscr{A}} \ln \lambda_{\mathfrak{z}}\right)^{n}(-1)^{k}(c)_{2 k}\left(\ln \lambda_{\mathfrak{z}}^{2}\right)^{k}}{n ! k !}
\end{aligned}
$$

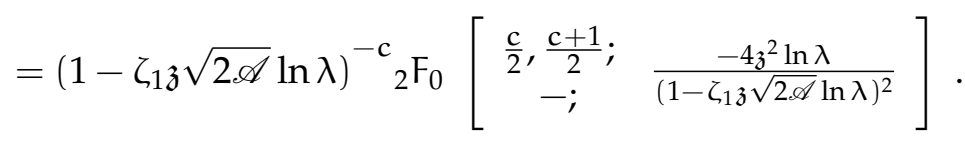

Hence the proof of (3.1).

Proff of (3.2).

$$
\sum_{n=0}^{\infty} \frac{M^{\mathscr{H}_{n}}\left(\zeta_{1} ; \lambda ; \mathscr{A}\right)_{M} \mathscr{H}_{\mathrm{n}}(y ; \lambda ; \mathscr{A}) \mathfrak{z}^{\mathrm{n}}}{\mathrm{n} !}=\sum_{n=0}^{\infty} \sum_{\mathrm{k}=0}^{[\mathrm{n} / 2]} \frac{(-1)^{\mathrm{k}}\left(\zeta_{1} \sqrt{2 \mathscr{A}}\right)^{\mathrm{n}-2 \mathrm{k}}(\ln \lambda)^{\mathrm{n}-\mathrm{k}}{ }^{\mathrm{M}} \mathscr{H}_{\mathrm{n}}(\mathrm{y} ; \lambda ; \mathscr{A}) \mathfrak{z}^{\mathrm{n}}}{\mathrm{k} !(\mathrm{n}-2 \mathrm{k}) !}
$$




$$
\begin{aligned}
& =\sum_{n=0}^{\infty} \sum_{k=0}^{\infty} \frac{(-1)^{\mathrm{k}}\left(\zeta_{1} \sqrt{2 \mathscr{A}}\right)^{\mathrm{n}}(\ln \lambda)^{\mathrm{n}+\mathrm{k}}{ }_{M} \mathscr{H}_{\mathrm{n}+2 \mathrm{k}}\left(\zeta_{2} ; \lambda ; \mathscr{A}\right) \mathfrak{z}^{\mathrm{n}+2 \mathrm{k}}}{\mathrm{k} ! \mathrm{n} !} \\
& =\sum_{n=0}^{\infty} \sum_{\mathrm{k}=0}^{\infty} \frac{\left(\zeta_{1} \sqrt{2 \mathscr{A}_{\mathfrak{z}}} \ln \lambda\right)^{\mathrm{n}}{ }_{M} \mathscr{H}_{\mathrm{n}+2 \mathrm{k}}(y ; \lambda ; \mathscr{A})(-1)^{\mathrm{k}}(\ln \lambda)^{\mathrm{k}} \mathfrak{z}^{2 \mathrm{k}}}{\mathrm{n} ! \mathrm{k} !}
\end{aligned}
$$

Now using the equation (3.3), we have

$$
\begin{aligned}
& =\sum_{k=0}^{\infty} \frac{\lambda^{\left(2 \mathscr{A} \zeta_{1} \zeta_{2} \mathfrak{z} \ln \lambda-2 \mathscr{A} \zeta_{1}^{2} \mathfrak{z}^{2}(\ln \lambda)^{2}\right)} M^{2} \mathscr{H}_{2 k}\left(\zeta_{2}-2 \zeta_{1} \mathfrak{z} \ln \lambda ; \lambda ; \mathscr{A}\right)(-1)^{k} \mathfrak{z}^{2 \mathrm{k}}(\ln \lambda)^{\mathrm{k}}}{\mathrm{k} !} \\
& =\left(1-4 \mathfrak{z}^{2} \ln \lambda^{2}\right)^{-\frac{1}{2}} \lambda^{\left(2 \mathscr{A} \zeta_{1} \zeta_{2} \mathfrak{z} \ln \lambda-2 \mathscr{A} \zeta_{1}^{2} \mathfrak{z}^{2}(\ln \lambda)^{2}\right)} \lambda^{\frac{\mathfrak{z}^{2} \ln \lambda^{2}\left(\zeta_{2} \sqrt{2 \mathscr{A}}-2 \zeta_{1} \mathfrak{z} \sqrt{2 \mathscr{A}} \ln \lambda\right)^{2}}{\left(1-4 \mathfrak{z}^{2} \ln \lambda^{2}\right)}} \\
& =\left(1-4 \mathfrak{z}^{2} \ln \lambda^{2}\right)^{-\frac{1}{2}} \lambda^{\frac{2 \mathscr{A} \mathfrak{z} \ln \lambda\left(\zeta_{1} \zeta_{2}-\zeta_{1}^{2} \mathfrak{z} \ln \lambda-\zeta_{2}^{2} \mathfrak{z}\right)}{\left(1-4_{\mathfrak{z}} 2 \ln \lambda^{2}\right)}} .
\end{aligned}
$$

Hence, we obtain the result of (3.2).

Proof of (3.3).

$$
\begin{aligned}
& \sum_{k=0}^{\infty} \sum_{n=0}^{\infty} \frac{M^{\mathscr{H}_{n+k}}\left(\zeta_{1} ; \lambda ; \mathscr{A}\right) \mathfrak{z}^{n} v^{k}}{n ! k !}=\sum_{n=0}^{\infty} \sum_{k=0}^{n} \frac{M^{\mathscr{H}_{n}}\left(\zeta_{1} ; \lambda ; \mathscr{A}\right) \mathfrak{z}^{n-k} v^{k}}{(n-k) ! k !} \\
& =\sum_{\mathrm{n}=0}^{\infty} \frac{\mathrm{M}_{\mathrm{n}}\left(\zeta_{1} ; \lambda ; \mathscr{A}\right)(\mathfrak{z}+v)^{\mathrm{n}}}{\mathrm{n} !} \\
& =\lambda^{\left[\zeta_{1}(\mathfrak{z}+v) \sqrt{2 \mathscr{A}}-(\mathfrak{z}+v)^{2} \mathrm{I}\right]} \\
& =\lambda^{\zeta_{1} \mathfrak{z} \sqrt{2 \mathscr{A}}-\mathfrak{z}^{2} I} \sum_{k=0}^{\infty} \frac{M^{\mathscr{H}_{k}}\left(\zeta_{1}-\left(2 \mathscr{A}^{-1}\right)^{\frac{1}{2}} \mathfrak{z} ; \lambda ; \mathscr{A}\right) v^{\mathrm{k}}}{\mathrm{k} !} \text {. }
\end{aligned}
$$

By equating the coefficients of $\frac{v^{k}}{k !}$ we have,

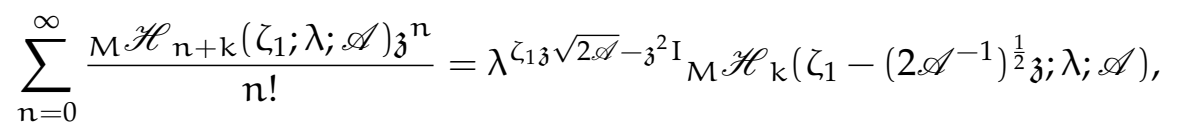

therefor the result.

Proof of (3.4). When applying equation (3.3) to any known generating relation and obtain a new result.

$$
\begin{aligned}
\sum_{k=0}^{\infty} \frac{(\mathrm{c})_{\mathrm{kM}} \mathscr{H}_{\mathrm{k}}\left(\zeta_{1}-\left(2 \mathscr{A}^{-1}\right)^{\frac{1}{2}} \mathfrak{z} ; \lambda ; \mathscr{A}\right)(-\mathfrak{z} y)^{\mathrm{k}}}{\mathrm{k} !} & =\sum_{\mathrm{k}=0}^{\infty} \sum_{\mathrm{n}=0}^{\infty} \frac{(\mathrm{c})_{\mathrm{k}} \lambda^{-\zeta_{1} \mathfrak{z} \sqrt{2 \mathscr{A}}+\mathfrak{z}^{2} \mathrm{I}}{ }_{M} \mathscr{H}_{\mathrm{n}+\mathrm{k}}\left(\zeta_{1} ; \lambda ; \mathscr{A}\right)(-\mathfrak{z} y)^{\mathrm{k}} \mathfrak{z}^{\mathrm{n}}}{\mathrm{n} ! \mathrm{k} !} \\
& =\lambda^{-\zeta_{1} \mathfrak{z} \sqrt{2 \mathscr{A}}+\mathfrak{z}^{2} \mathrm{I}} \sum_{n=0}^{\infty} \sum_{\mathrm{k}=0}^{\mathrm{n}} \frac{(-1)^{\mathrm{k}}(\mathrm{c})_{\mathrm{k}} \zeta_{2}^{\mathrm{k}}{ } \mathscr{H}_{\mathrm{n}}\left(\zeta_{1} ; \lambda ; \mathscr{A}\right) \mathfrak{z}^{\mathrm{n}}}{\mathrm{k} !(\mathrm{n}-\mathrm{k}) !} \\
& =\lambda^{-\zeta_{1 \mathfrak{z}} \sqrt{2 \mathscr{A}}+\mathfrak{z}^{2} \mathrm{I}} \sum_{\mathrm{n}=0}^{\infty} \frac{{ }_{2} \mathrm{~F}_{0}\left(-\mathrm{n}, \mathrm{c} ;-; \zeta_{2}\right)_{\mathrm{M}} \mathscr{H}_{\mathrm{n}}\left(\zeta_{1} ; \lambda ; \mathscr{A}\right) \mathfrak{z}^{\mathrm{n}}}{\mathrm{n} !}
\end{aligned}
$$

using (3.1), then

$$
\sum_{n=0}^{\infty} \frac{{ }_{2} F_{0}\left[-n, c ;-; \zeta_{2}\right]_{M} \mathscr{H}_{\mathrm{n}}\left(\zeta_{1} ; \lambda ; \mathscr{A}\right) \mathfrak{z}^{\mathrm{n}}}{n !}=\lambda^{\zeta_{1} \mathfrak{z} \sqrt{2 \mathscr{A}}-\mathfrak{z}^{2} \mathrm{I}}\left(1+\zeta_{2} \mathfrak{z} \sqrt{2 \mathscr{A}}\left(\zeta_{1}-\sqrt{2 \mathscr{A}^{-1}}\right)\right)^{-\mathrm{c}}
$$




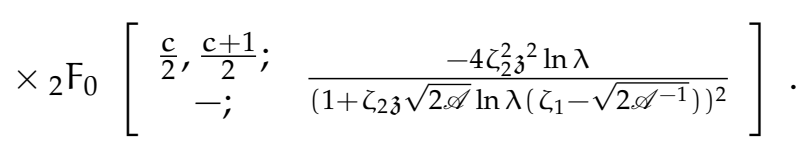

Hence proved.

Proof of (3.5).

$$
\begin{aligned}
& \sum_{n=0}^{\infty} \frac{(-1)^{n}{ }_{M} \mathscr{H}_{2 n}\left(\zeta_{1} ; \lambda ; \mathscr{A}\right) \mathfrak{z}^{n}}{(2 n) !}=\sum_{n=0}^{\infty} \sum_{k=0}^{n} \frac{(-1)^{n+k}\left(\zeta_{1} \sqrt{2 \mathscr{A}}\right)^{2 n-2 k}(\ln \lambda)^{2 n-k} \mathfrak{z}^{n}}{k !(2 n-2 k) !} \\
& =\sum_{n=0}^{\infty} \sum_{k=0}^{\infty} \frac{(-1)^{n+2 k}\left(\zeta_{1} \sqrt{2 \mathscr{A}}\right)^{2 n}(\ln \lambda)^{2 n+k} \mathfrak{z}^{n+k}}{k !(2 n) !} \\
& =\lambda^{\mathfrak{z}} \cos \left(\zeta_{1} \ln \lambda \sqrt{2 \mathscr{A} \mathfrak{z}}\right) \text {. }
\end{aligned}
$$

Similarly, we can prove the other results.

\section{Recurrence relations of ${ }_{M} \mathscr{H}_{\mathbf{n}}\left(\zeta_{1} ; \lambda ; \mathscr{A}\right)$}

The generating relation of ${ }_{M} \mathscr{H}_{\mathrm{n}}\left(\zeta_{1} ; \lambda ; \mathscr{A}\right)$ is

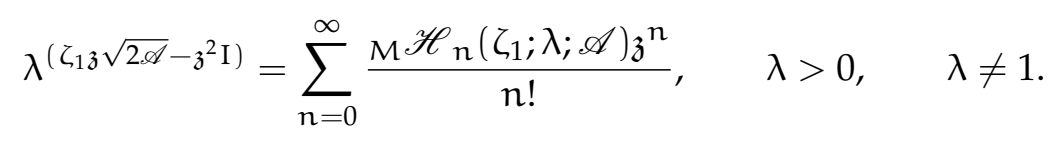

Let

$$
F=\lambda^{\left(\zeta_{1} \mathfrak{z} \sqrt{2 \mathscr{A}}-\mathfrak{z}^{2} I\right)}
$$

then

$$
\begin{aligned}
\frac{\partial \mathrm{F}}{\partial \zeta_{1}} & =\lambda^{\left(\zeta_{1} \mathfrak{z} \sqrt{2 \mathscr{A}}-\mathfrak{z}^{2} \mathrm{I}\right)}(\mathfrak{z} \sqrt{2 \mathscr{A}}) \ln \lambda, \\
\frac{\partial \mathrm{F}}{\partial \mathfrak{z}} & =\lambda^{\left(\zeta_{1} \mathfrak{z} \sqrt{2 \mathscr{A}}-\mathfrak{z}^{2} \mathrm{I}\right)}\left(\zeta_{1} \sqrt{2 \mathscr{A}}-2 \mathfrak{z} \mathrm{I}\right) \ln \lambda .
\end{aligned}
$$

Multiplying equation (4.1) by $\left(\zeta_{1} \sqrt{2 \mathscr{A}}-2 \mathfrak{z} I\right)$ and equation (4.2) by $(\mathfrak{z} \sqrt{2 \mathscr{A}})$ and subtracting

$$
\begin{aligned}
& \left(\zeta_{1} \sqrt{2 \mathscr{A}}-2 \mathfrak{z} \mathrm{I}\right) \frac{\partial \mathrm{F}}{\partial \zeta_{1}}-(\mathfrak{z} \sqrt{2 \mathscr{A}}) \frac{\partial \mathrm{F}}{\partial \mathfrak{z}}=0, \\
& \sum_{n=0}^{\infty} \frac{\zeta_{1} \sqrt{2 \mathscr{A}}_{M} \mathscr{H}_{n}{ }^{\prime}\left(\zeta_{1} ; \lambda ; \mathscr{A}\right) \mathfrak{z}^{n}}{n !}-\sum_{n=0}^{\infty} \frac{2_{M} \mathscr{H}_{n}{ }^{\prime}\left(\zeta_{1} ; \lambda ; \mathscr{A}\right) \mathfrak{z}^{n+1}}{n !}-\sum_{n=0}^{\infty} \frac{n \sqrt{2 \mathscr{A}_{M}} \mathscr{H}_{n}\left(\zeta_{1} ; \lambda ; \mathscr{A}\right) \mathfrak{z}^{n}}{n !}=0 .
\end{aligned}
$$

Comparing the coefficient of $\frac{\hat{z}^{\mathrm{n}}}{\mathrm{n} !}$ yields the recurrence relation

$$
\zeta_{1} \sqrt{2 \mathscr{A}}_{\mathrm{M}} \mathscr{H}_{\mathrm{n}}{ }^{\prime}\left(\zeta_{1} ; \lambda ; \mathscr{A}\right)-2 \mathrm{n}_{\mathrm{M}} \mathscr{H}_{\mathrm{n}-1}{ }^{\prime}\left(\zeta_{1} ; \lambda ; \mathscr{A}\right)=\mathrm{n} \sqrt{2 \mathscr{A}}_{\mathrm{M}} \mathscr{H}_{\mathrm{n}}\left(\zeta_{1} ; \lambda ; \mathscr{A}\right)
$$

Using $\frac{\partial F}{\partial \zeta_{1}}$

$$
\sum_{n=0}^{\infty} \frac{M^{\mathscr{H}_{n}}{ }^{\prime}\left(\zeta_{1} ; \lambda ; \mathscr{A}\right) \mathfrak{z}^{n}}{n !}=\sum_{n=0}^{\infty} \frac{\ln \lambda \sqrt{2 \mathscr{A}_{M}} \mathscr{H}_{\mathrm{n}}\left(\zeta_{1} ; \lambda ; \mathscr{A}\right) \mathfrak{z}^{n+1}}{n !}=\sum_{n=0}^{\infty} \frac{\mathrm{n} \ln \lambda \sqrt{2 \mathscr{A}}_{\mathrm{M}} \mathscr{H}_{\mathrm{n}-1}\left(\zeta_{1} ; \lambda ; \mathscr{A}\right) \mathfrak{z}^{\mathrm{n}}}{\mathrm{n} !}
$$

yields the recurrence relation

$$
\mathrm{M}^{\mathscr{H}_{\mathrm{n}}}{ }^{\prime}\left(\zeta_{1} ; \lambda ; \mathscr{A}\right)=\mathrm{n} \ln \lambda \sqrt{2 \mathscr{A}}_{\mathrm{M}} \mathscr{H}_{\mathrm{n}-1}\left(\zeta_{1} ; \lambda ; \mathscr{A}\right) .
$$


By equation (4.4) and (4.3) we have the recurrence relation

$$
2 \mathscr{A} \zeta_{1} \ln \lambda_{\mathrm{M}} \mathscr{H}_{\mathrm{n}-1}\left(\zeta_{1} ; \lambda ; \mathscr{A}\right)-2(\mathrm{n}-1) \sqrt{2 \mathscr{A}} \ln \lambda_{\mathrm{M}} \mathscr{H}_{\mathrm{n}-2}\left(\zeta_{1} ; \lambda ; \mathscr{A}\right)=\sqrt{2 \mathscr{A}}_{\mathrm{M}} \mathscr{H}_{\mathrm{n}}\left(\zeta_{1} ; \lambda ; \mathscr{A}\right) .
$$

Replacing $n$ by $n+1$ in (4.5), to obtain the three terms recurrence relations in the form

$$
{ }_{M} \mathscr{H}_{\mathrm{n}+1}\left(\zeta_{1} ; \lambda ; \mathscr{A}\right)=\sqrt{2 \mathscr{A}_{1}} \ln \lambda_{\mathrm{M}} \mathscr{H}_{\mathrm{n}}\left(\zeta_{1} ; \lambda ; \mathscr{A}\right)-2(\mathrm{n}) \ln \lambda_{\mathrm{M}} \mathscr{H}_{\mathrm{n}-1}\left(\zeta_{1} ; \lambda ; \mathscr{A}\right) .
$$

Also,

$$
\mathrm{M}^{\mathscr{H}_{\mathrm{n}}}{ }^{\prime \prime}\left(\zeta_{1} ; \lambda ; \mathscr{A}\right)=\mathrm{n}(\mathrm{n}-1)(\ln \lambda \sqrt{2 \mathscr{A}})^{2}{ }_{\mathrm{M}} \mathscr{H}_{\mathrm{n}-2}\left(\zeta_{1} ; \lambda ; \mathscr{A}\right),
$$

using (4.5) in (4.7) yields the modified Hermite matrix differential equation

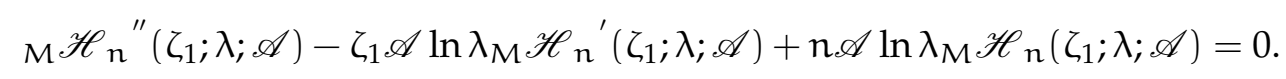

\section{Rodrigues formula for $\mathrm{M}_{\mathrm{H}} \mathscr{H}_{\mathbf{n}}\left(\zeta_{1} ; \lambda ; \mathscr{A}\right)$}

The Rodrigues formula for the modified Hermite matrix polynomials ${ }_{M} \mathscr{H}_{\mathrm{n}}\left(\zeta_{1} ; \lambda ; \mathscr{A}\right)$ is given by the following relation,

$$
\mathrm{M}^{\mathscr{H}_{\mathrm{n}}}\left(\zeta_{1} ; \lambda ; \mathscr{A}\right)=(-1)^{\mathrm{n}}\left(\frac{\mathscr{A}}{2}\right)^{-\frac{\mathrm{n}}{2}} \lambda^{\frac{\zeta_{1}^{2} \mathscr{A}}{2}} \mathrm{D}^{\mathrm{n}}\left(\lambda^{-\frac{\zeta_{1}^{2} \mathscr{A}}{2}}\right) .
$$

The proof of (5.1) is as

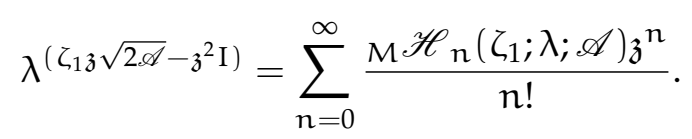

Using the Maclaurin's theorem

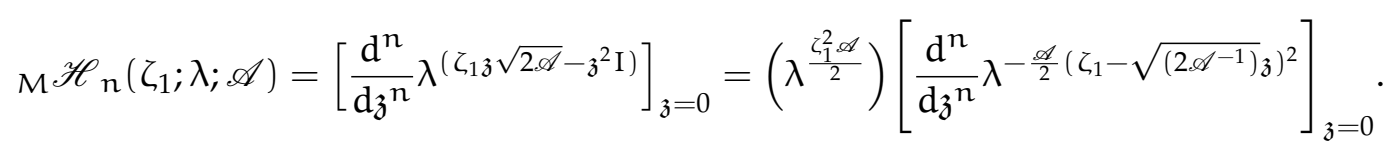

Now put $\left(\zeta_{1}-\sqrt{\left(2 \mathscr{A}^{-1}\right)} \mathfrak{z}\right)=w$,

$$
=\left(\lambda^{\frac{\zeta_{1}^{2} \mathscr{A}}{2}}\right)(-1)^{\mathrm{n}}\left(\frac{\mathscr{A}}{2}\right)^{-\frac{\mathrm{n}}{2}}\left[\frac{\mathrm{d}^{\mathrm{n}}}{\mathrm{d} w^{\mathrm{n}}} \lambda^{-\frac{\mathscr{A}}{2} w^{2}}\right]_{w=\zeta_{1}},
$$

when $w=\zeta_{1}$, where $\frac{d}{d \zeta_{1}}=D$,

$$
\mathrm{M}^{\mathscr{H}_{\mathrm{n}}}\left(\zeta_{1} ; \lambda ; \mathscr{A}\right)=(-1)^{\mathrm{n}}\left(\frac{\mathscr{A}}{2}\right)^{-\frac{\mathrm{n}}{2}} \lambda^{\frac{\zeta_{1}^{2} \mathscr{A}}{2}} \mathrm{D}^{\mathrm{n}}\left(\lambda^{-\frac{\zeta_{1}^{2} \mathscr{A}}{2}}\right) .
$$

Hence the proof of Rodrigues formula.

6. Hypergeometric form of ${ }_{\mathrm{M}} \mathscr{H}_{\mathbf{n}}\left(\zeta_{1} ; \lambda ; \mathscr{A}\right)$

$$
\begin{aligned}
& M \mathscr{H}_{\mathrm{n}}\left(\zeta_{1} ; \lambda ; \mathscr{A}\right)=\sum_{\mathrm{k}=0}^{[\mathrm{n} / 2]} \frac{(-1)^{\mathrm{k}} \mathrm{n} !\left(\zeta_{1} \sqrt{2 \mathscr{A}}\right)^{\mathrm{n}-2 \mathrm{k}}(\ln \lambda)^{\mathrm{n}-\mathrm{k}}}{\mathrm{k} !(\mathrm{n}-2 \mathrm{k}) !} \\
& =\sum_{k=0}^{[n / 2]} \frac{(-1)^{k}(-n)_{2 k}\left(\zeta_{1} \sqrt{2 \mathscr{A}}\right)^{n-2 k}(\ln \lambda)^{n-k}}{k !}
\end{aligned}
$$

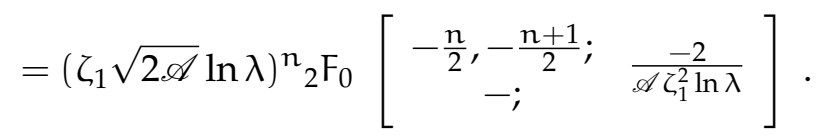




\section{Orthogonality of ${ }_{\mathrm{M}} \mathscr{H}_{\mathbf{n}}\left(\zeta_{1} ; \lambda ; \mathscr{A}\right)$}

The differential equation of ${ }_{\mathrm{M}} \mathscr{H}_{\mathrm{n}}\left(\zeta_{1} ; \lambda ; \mathscr{A}\right)$ is given by equation (4.8),

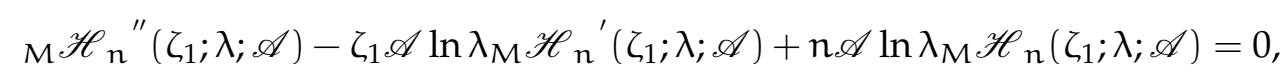

which may be written as

$$
\left(\lambda^{-\frac{\zeta_{1}^{2} \mathscr{A}}{2}} \mathrm{M} \mathscr{H}_{\mathrm{n}}{ }^{\prime}\left(\zeta_{1} ; \lambda ; \mathscr{A}\right)\right)^{\prime}+\mathrm{n} \mathscr{A} \ln \lambda\left(\lambda^{-\frac{\zeta_{1}^{2} \mathscr{A}}{2}}\right){ }_{\mathrm{M}} \mathscr{H}_{\mathrm{n}}\left(\zeta_{1} ; \lambda ; \mathscr{A}\right)=0
$$

Along with above equation (7.1) write

$$
\left(\lambda^{-\frac{\zeta_{1}^{2} \mathscr{A}}{2}} \mathrm{M}^{\mathscr{H}_{\mathrm{m}}}{ }^{\prime}\left(\zeta_{1} ; \lambda ; \mathscr{A}\right)\right)^{\prime}+\mathrm{m} \mathscr{A} \ln \lambda\left(\lambda^{-\frac{\zeta_{1}^{2} \mathscr{A}}{2}}\right) \mathrm{M} \mathscr{H}_{\mathrm{n}}\left(\zeta_{1} ; \lambda ; \mathscr{A}\right)=0 .
$$

Combining (7.1) and (7.2), we obtain

$$
\begin{aligned}
(\mathrm{n}-\mathrm{m}) & \mathscr{A} \ln \lambda\left(\lambda^{-\frac{\zeta_{1}^{2} \mathscr{A}}{2}}\right){ }_{\mathrm{M}} \mathscr{H}_{\mathrm{n}}\left(\zeta_{1} ; \lambda ; \mathscr{A}\right)_{\mathrm{M}} \mathscr{H}_{\mathrm{m}}\left(\zeta_{1} ; \lambda ; \mathscr{A}\right) \\
= & {\left[\lambda^{-\frac{\zeta_{1}^{2} \mathscr{A}}{2}}\left({ }_{\mathrm{M}} \mathscr{H}_{\mathrm{n}}\left(\zeta_{1} ; \lambda ; \mathscr{A}\right)_{\mathrm{M}} \mathscr{H}_{\mathrm{m}}{ }^{\prime}\left(\zeta_{1} ; \lambda ; \mathscr{A}\right)-{ }_{\mathrm{M}} \mathscr{H}_{\mathrm{n}}{ }^{\prime}\left(\zeta_{1} ; \lambda ; \mathscr{A}\right)_{\mathrm{M}} \mathscr{H}_{\mathrm{m}}\left(\zeta_{1} ; \lambda ; \mathscr{A}\right)\right)\right]^{\prime} . }
\end{aligned}
$$

It follows that

$$
\begin{aligned}
(\mathrm{n}-\mathrm{m}) & \mathscr{A} \ln \lambda \int_{\mathrm{a}}^{\mathrm{b}}\left(\lambda^{-\frac{\zeta_{1}^{2} \mathscr{A}}{2}}\right)_{\mathrm{M}} \mathscr{H}_{\mathrm{n}}\left(\zeta_{1} ; \lambda ; \mathscr{A}\right)_{\mathrm{M}} \mathscr{H}_{\mathrm{m}}\left(\zeta_{1} ; \lambda ; \mathscr{A}\right) \mathrm{d} \zeta_{1} \\
= & {\left[\lambda^{-\frac{\zeta_{1}^{2} \mathscr{A}}{2}}\left(\mathrm{M}_{\mathrm{M}} \mathscr{H}_{\mathrm{n}}\left(\zeta_{1} ; \lambda ; \mathscr{A}\right)_{\mathrm{M}} \mathscr{H}_{\mathrm{m}}{ }^{\prime}\left(\zeta_{1} ; \lambda ; \mathscr{A}\right)-\mathrm{M}_{\mathrm{M}} \mathscr{H}_{\mathrm{n}}{ }^{\prime}\left(\zeta_{1} ; \lambda ; \mathscr{A}\right)_{\mathrm{M}} \mathscr{H}_{\mathrm{m}}\left(\zeta_{1} ; \lambda ; \mathscr{A}\right)\right)\right]_{\mathrm{a}}^{\mathrm{b}} . }
\end{aligned}
$$

Since the product of any polynomials in $\zeta_{1}$ by $\lambda^{-\frac{\zeta_{1}^{2} \mathscr{A}}{2}} \rightarrow 0$ as $\zeta_{1} \rightarrow \infty$ or $\zeta_{1} \rightarrow-\infty$, we may conclude that

$$
\int_{-\infty}^{\infty}\left(\lambda^{-\frac{\zeta_{1}^{2} \mathscr{A}}{2}}\right)_{\mathrm{M}} \mathscr{H}_{\mathrm{n}}\left(\zeta_{1} ; \lambda ; \mathscr{A}\right)_{\mathrm{M}} \mathscr{H}_{\mathrm{m}}\left(\zeta_{1} ; \lambda ; \mathscr{A}\right) \mathrm{d} \zeta_{1}=0 ; \quad \text { for } \quad \mathrm{m} \neq \mathrm{n} .
$$

Now for $m=n$ we have

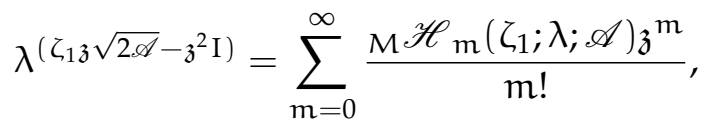

$$
\begin{aligned}
& \lambda^{\left(2 \zeta_{1} \mathfrak{z} \sqrt{2 \mathscr{A}}-2 \mathfrak{z}^{2} \mathrm{I}\right)}=\sum_{\mathrm{m}=0}^{\infty} \sum_{\mathrm{k}=0}^{\infty} \frac{\mathrm{M}^{\mathscr{H}_{\mathrm{m}}}\left(\zeta_{1} ; \lambda ; \mathscr{A}\right)_{\mathrm{M}} \mathscr{H}_{\mathrm{k}}\left(\zeta_{1} ; \lambda ; \mathscr{A}\right) \mathfrak{z}^{\mathrm{m}+\mathrm{k}}}{\mathrm{m} ! \mathrm{k} !}
\end{aligned}
$$

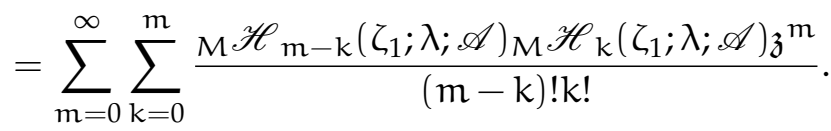

$$
\begin{aligned}
& \int_{-\infty}^{\infty} \lambda^{\left(2 \zeta_{1} \mathfrak{z} \sqrt{2 \mathscr{A}}-2 \mathfrak{z}^{2} \mathrm{I}-\frac{\zeta_{1}^{2} \mathscr{A}}{2}\right)} \mathrm{d} \zeta_{1}=\sum_{\mathrm{m}=0}^{\infty} \sum_{\mathrm{k}=0}^{\mathrm{m}} \int_{-\infty}^{\infty} \frac{\lambda^{-\frac{\zeta_{1}^{2} \mathscr{A}}{2}} \mathrm{M} \mathscr{H}_{\mathrm{m}-\mathrm{k}}\left(\zeta_{1} ; \lambda ; \mathscr{A}\right)_{\mathrm{M}} \mathscr{H}_{\mathrm{k}}\left(\zeta_{1} ; \lambda ; \mathscr{A}\right) \mathfrak{z}^{\mathrm{m}}}{(\mathrm{m}-\mathrm{k}) ! \mathrm{k} !} \mathrm{d} \zeta_{1}, \\
& \lambda^{2 \mathfrak{z}^{2} \mathrm{I}} \int_{-\infty}^{\infty} \lambda^{\left(2 \zeta_{1} \mathfrak{z} \sqrt{2 \mathscr{A}}-4 \mathfrak{z}^{2} \mathrm{I}-\frac{\zeta_{1}^{2} \mathscr{A}}{2}\right)} \mathrm{d} \zeta_{1}=\sum_{n=0}^{\infty} \int_{-\infty}^{\infty} \frac{\lambda^{-\frac{\zeta_{1}^{2} \mathscr{A}}{2}} \mathrm{M} \mathscr{H}_{\mathrm{n}}{ }^{2}\left(\zeta_{1} ; \lambda ; \mathscr{A}\right) \mathfrak{z}^{2 \mathrm{n}}}{(\mathrm{n})^{2} !} \mathrm{d} \zeta_{1}, \\
& \lambda^{2 \mathfrak{z}^{2} \mathrm{I}} \int_{-\infty}^{\infty} \lambda^{\frac{\mathscr{A}}{2}\left(\zeta_{1} \mathrm{I}-2 \sqrt{2 \mathscr{A}^{-1}} \mathfrak{z}\right)^{2}} \mathrm{~d} \zeta_{1}=\sum_{n=0}^{\infty} \int_{-\infty}^{\infty} \frac{\lambda^{-\frac{\zeta_{1}^{2} \mathscr{A}}{2}} \mathrm{M} \mathscr{H}_{\mathrm{n}}{ }^{2}\left(\zeta_{1} ; \lambda ; \mathscr{A}\right) \mathfrak{z}^{2 \mathrm{n}}}{(\mathrm{n})^{2} !} \mathrm{d} \zeta_{1}
\end{aligned}
$$




$$
\sqrt{\frac{2 \pi \mathscr{A}^{-1}}{\ln \lambda}} \sum_{n=0}^{\infty} \frac{2^{\mathrm{n}}(\ln \lambda)^{\mathrm{n}} \mathfrak{z}^{2 \mathrm{n}}}{\mathrm{n} !}=\sum_{\mathrm{n}=0}^{\infty} \int_{-\infty}^{\infty} \frac{\lambda^{-\frac{\zeta_{1}^{2} \mathscr{A}}{2}} M^{\mathscr{H}_{n}}{ }^{2}\left(\zeta_{1} ; \lambda ; \mathscr{A}\right) \mathfrak{z}^{2 \mathrm{n}}}{(\mathrm{n})^{2} !} \mathrm{d} \zeta_{1} .
$$

So

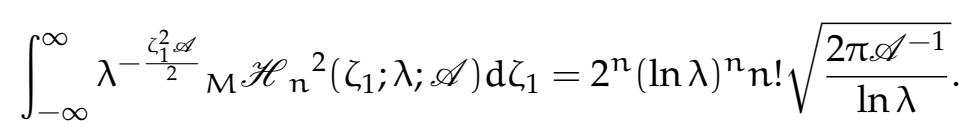

Hence, the orthogonality condition for Modified Hermite Matrix polynomials $\mathrm{M}_{\mathrm{H}} \mathscr{H}_{\mathrm{n}}\left(\zeta_{1} ; \lambda ; \mathscr{A}\right)$ is as follows

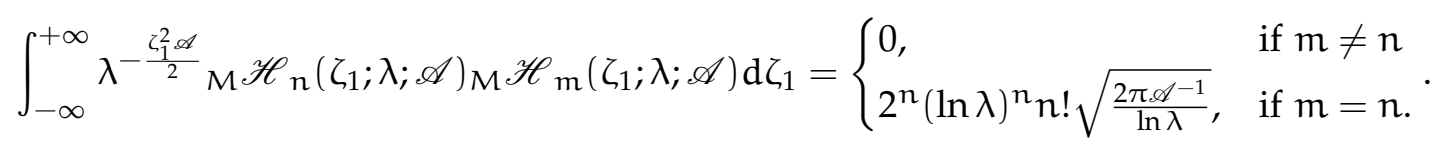

Theorem 7.1. For the modified Hermite matrix polynomials $\mathrm{M}_{\mathrm{H}}\left(\zeta_{1} ; \lambda ; \mathscr{A}\right)$,

(a)

$$
\int_{-\infty}^{+\infty} \lambda^{-\frac{\zeta_{1}^{2} \mathscr{A}}{2}} \zeta_{1}^{\mathrm{k}} \mathrm{M} \mathscr{H}_{\mathrm{n}}\left(\zeta_{1} ; \lambda ; \mathscr{A}\right) \mathrm{d} \zeta_{1}=0 ; \quad \text { for } \mathrm{k}=0,1,2, \cdots(\mathrm{n}-1) ;
$$

(b) the zeros of $\mathrm{M} \mathscr{H}_{\mathrm{n}}\left(\zeta_{1} ; \lambda ; \mathscr{A}\right)$ are real and distinct;

(c) the Christoffel-Darboux formula of summation is

$$
\begin{aligned}
& \sum_{\mathrm{k}=0}^{\mathrm{n}} \frac{{ }^{\mathrm{M}} \mathscr{H}_{\mathrm{k}}\left(\zeta_{1} ; \lambda ; \mathscr{A}\right)_{\mathrm{M}} \mathscr{H}_{\mathrm{k}}\left(\zeta_{1} ; \lambda ; \mathscr{A}\right)}{\mathrm{k} ! 2^{\mathrm{k}}(\ln \lambda)^{\mathrm{k}}}
\end{aligned}
$$

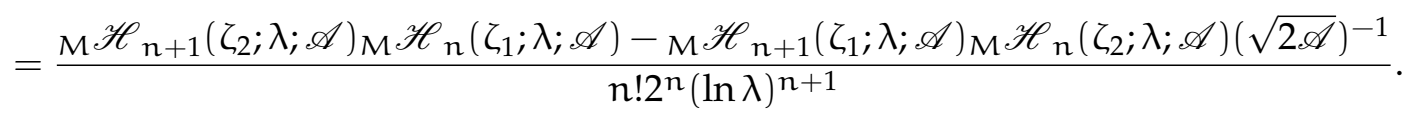

Proof. The proof of parts (a) and (b) are straightforward. Hence we omit the details.

Proof of (c): Using the equation (4.6)

$$
\mathrm{M}^{\mathscr{H}_{\mathrm{k}+1}}\left(\zeta_{1} ; \lambda ; \mathscr{A}\right)=\sqrt{2 \mathscr{A}} \zeta_{1} \ln \lambda_{\mathrm{M}} \mathscr{H}_{\mathrm{k}}\left(\zeta_{1} ; \lambda ; \mathscr{A}\right)-2(\mathrm{k}) \ln \lambda_{\mathrm{M}} \mathscr{H}_{\mathrm{k}-1}\left(\zeta_{1} ; \lambda ; \mathscr{A}\right)
$$

Now, multiplying by $\frac{\mathrm{M} \mathscr{H}_{\mathrm{k}}\left(\zeta_{2} ; \lambda ; \mathscr{A}\right)}{\mathrm{k} ! 2^{\mathrm{k}+1}}$, yield the equation

$$
\begin{aligned}
& \frac{\mathrm{M} \mathscr{H}_{\mathrm{k}+1}\left(\zeta_{1} ; \lambda ; \mathscr{A}\right)_{\mathrm{M}} \mathscr{H}_{\mathrm{k}}\left(\zeta_{2} ; \lambda ; \mathscr{A}\right)}{\mathrm{k} ! 2^{\mathrm{k}+1}}=\sqrt{2 \mathscr{A}} \zeta_{1} \ln \lambda \frac{{ }^{\mathrm{M}} \mathscr{H}_{\mathrm{k}}\left(\zeta_{1} ; \lambda ; \mathscr{A}\right)_{\mathrm{M}} \mathscr{H}_{\mathrm{k}}\left(\zeta_{2} ; \lambda ; \mathscr{A}\right)}{\mathrm{k} ! 2^{\mathrm{k}+1}}
\end{aligned}
$$

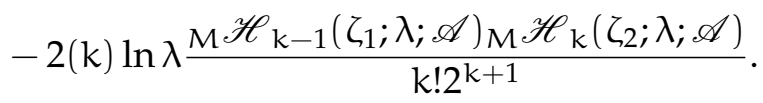

Interchanging $\zeta_{1}$ and $\zeta_{2}$, yield the equation

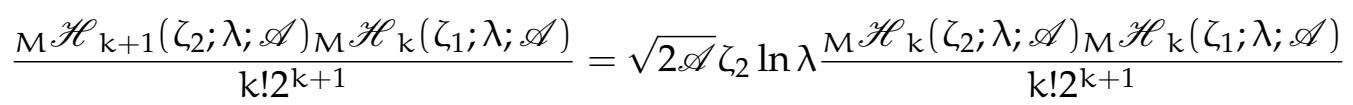

$$
\begin{aligned}
& -2(\mathrm{k}) \ln \lambda \frac{\mathrm{M} \mathscr{H}_{\mathrm{k}-1}\left(\zeta_{2} ; \lambda ; \mathscr{A}\right)_{\mathrm{M}} \mathscr{H}_{\mathrm{k}}\left(\zeta_{1} ; \lambda ; \mathscr{A}\right)}{\mathrm{k} ! 2^{\mathrm{k}+1}} .
\end{aligned}
$$

Subtracting the equation (7.3) from (7.4) and putting $k=0,1,2, \ldots, n$, yields the result.

\section{Expansion of polynomials}

Any polynomials can be expended in a series of modified Hermite matrix polynomials and the coefficients can be determined as

$$
\zeta_{1}^{n}=\sum_{k=0}^{[n / 2]} \frac{n !(2 \mathscr{A})^{-n / 2} M_{M} \mathscr{H}_{n-2 k}\left(\zeta_{1} ; \lambda ; \mathscr{A}\right)(\ln \lambda)^{k-n}}{k !(n-2 k) !} .
$$


Expansion of the Legender matrix polynomials in a series of ${ }_{\mathrm{M}} \mathscr{H}_{\mathrm{n}}\left(\zeta_{1} ; \lambda ; \mathscr{A}\right)$ is

$$
\mathscr{P}_{\mathrm{n}}\left(\zeta_{1}, \mathscr{A}\right)=\sum_{\mathrm{k}=0}^{[\mathrm{n} / 2]} \frac{(-1)^{\mathrm{k}}\left(\frac{1}{2}\right)_{\mathrm{n}-\mathrm{k}}(\ln \lambda)^{2 \mathrm{k}-\mathrm{n}} \mathrm{M} \mathscr{H}_{\mathrm{n}-2 \mathrm{k}}\left(\zeta_{1} ; \lambda ; \mathscr{A}\right)}{\mathrm{k} !(\mathrm{n}-2 \mathrm{k}) !}{ }_{2} \mathrm{~F}_{0}\left[-\mathrm{k}, \frac{1}{2}+\mathrm{n}-\mathrm{k} ;-; \frac{1}{\ln \lambda}\right] .
$$

Expansion of the modified Hermite matrix polynomials in a series of Legender matrix polynomials is

$$
M \mathscr{H}_{\mathrm{n}}\left(\zeta_{1} ; \lambda ; \mathscr{A}\right)=\sum_{\mathrm{k}=0}^{[\mathrm{n} / 2]} \frac{(-1)^{\mathrm{k}} \mathrm{n} !(2 \mathrm{n}-4 \mathrm{k}+1)(\ln \lambda)^{\mathrm{n}-\mathrm{k}} \mathscr{P}_{\mathrm{n}-2 \mathrm{k}}\left(\zeta_{1}, \mathscr{A}\right)}{\mathrm{k} !\left(\frac{3}{2}\right)_{n-2 k}}{ }_{1} \mathrm{~F}_{1}\left[-\mathrm{k} ; \frac{3}{2}+\mathrm{n}-2 \mathrm{k} ; \ln \lambda\right] .
$$

Expansion of modified Hermite matrix polynomials in a series of Laguerre matrix polynomials is

$$
\begin{aligned}
& \mathrm{M} \mathscr{H}_{\mathrm{n}}\left(\zeta_{1} ; \lambda ; \mathscr{A}\right)=(\mathscr{A}+\mathrm{I})_{\mathrm{n}}(\sqrt{2 \mathscr{A}} \ln \lambda)^{\mathrm{n}} \sum_{\mathrm{s}=0}^{\mathrm{n}}(-\mathrm{n})_{\mathrm{s}}\left[(\mathscr{A}+\mathrm{I})_{\mathrm{s}}\right]^{-1} \\
& \times{ }_{2} \mathrm{~F}_{2}\left[\begin{array}{cc}
-\frac{(\mathrm{n}-\mathrm{s}) \mathrm{I}}{2},-\frac{(\mathrm{n}-\mathrm{s}-1) \mathrm{I}}{2} ; & -\frac{1}{2 \mathscr{A} \ln \lambda}
\end{array}\right] \mathrm{L}_{\mathrm{s}}^{\mathscr{A}}\left(\zeta_{1}\right) .
\end{aligned}
$$

Proof of (8.2). Consider the series of Legender matrix polynomials form [17, 30, 33],

$$
\sum_{n=0}^{\infty} \mathscr{P}_{n}\left(\zeta_{1}, \mathscr{A}\right) \mathfrak{z}^{n}=\sum_{n=0}^{\infty} \sum_{k=0}^{[n / 2]} \frac{(-1)^{k}\left(\frac{1}{2}\right)_{n-k}\left(\zeta_{1} \sqrt{2 \mathscr{A}}\right)^{n-2 k} \mathfrak{z}^{n}}{k !(n-2 k) !}
$$

Using equation (8.1), we may write

$$
\begin{aligned}
& \sum_{n=0}^{\infty} \mathscr{P}_{n}\left(\zeta_{1}, \mathscr{A}\right) \mathfrak{z}^{n}=\sum_{n=0}^{\infty} \sum_{k=0}^{\infty} \sum_{s=0}^{[n / 2]} \frac{(-1)^{k}\left(\frac{1}{2}\right)_{n+k M} \mathscr{H}_{n-2 s}\left(\zeta_{1} ; \lambda ; \mathscr{A}\right)(\ln \lambda)^{s-n_{\mathfrak{z}} n+2 k}}{k ! s !(n-2 k) !} \\
& =\sum_{n=0}^{\infty} \sum_{k=0}^{\infty} \sum_{s=0}^{k} \frac{(-1)^{k-s}\left(\frac{1}{2}\right)_{n+k+s M} \mathscr{H}_{n}\left(\zeta_{1} ; \lambda ; \mathscr{A}\right)(\ln \lambda)^{-(n+s)} \mathfrak{z}^{n+2 k}}{(k-s) ! s ! n !} \\
& =\sum_{n=0}^{\infty} \sum_{k=0}^{\infty} \frac{(-1)^{k}\left(\frac{1}{2}\right)_{n+k M} \mathscr{H}_{n}\left(\zeta_{1} ; \lambda ; \mathscr{A}\right)(\ln \lambda)^{-(n)} \mathfrak{z}^{n+2 k}}{k ! n !}{ }_{2} F_{0}\left[-k, \frac{1}{2}+n+k ;-; \frac{1}{\ln \lambda}\right] \text {. }
\end{aligned}
$$

Using the equation (1.1),

$$
=\sum_{n=0}^{\infty} \sum_{k=0}^{[n / 2]} \frac{(-1)^{k}\left(\frac{1}{2}\right)_{n-k M} \mathscr{H}_{n-2 k}\left(\zeta_{1} ; \lambda ; \mathscr{A}\right)(\ln \lambda)^{-n+2 k} \mathfrak{z}^{n}}{k !(n-2 k) !}{ }_{2} F_{0}\left[-k, \frac{1}{2}+n+k ;-; \frac{1}{\ln \lambda}\right] .
$$

Hence, the final result is

$$
\mathscr{P}_{\mathrm{n}}\left(\zeta_{1}, \mathscr{A}\right)=\sum_{\mathrm{k}=0}^{[\mathrm{n} / 2]} \frac{(-1)^{\mathrm{k}}\left(\frac{1}{2}\right)_{\mathrm{n}-\mathrm{k}}(\ln \lambda)^{2 \mathrm{k}-\mathrm{n}} \mathrm{M} \mathscr{H}_{\mathrm{n}-2 \mathrm{k}}\left(\zeta_{1} ; \lambda ; \mathscr{A}\right)}{\mathrm{k} !(\mathrm{n}-2 \mathrm{k}) !}{ }_{2} \mathrm{~F}_{0}\left[-\mathrm{k}, \frac{1}{2}+\mathrm{n}-\mathrm{k} ;-; \frac{1}{\ln \lambda}\right] .
$$

Proof of (8.3). Now, from $[8,17,30]$ we have

$$
\frac{\left(\zeta_{1} \sqrt{2 \mathscr{A}}\right)^{n}}{n !}=\sum_{k=0}^{[n / 2]} \frac{(2 n-4 k+1) \mathscr{P}_{n-2 k}\left(\zeta_{1}, \mathscr{A}\right)}{k !\left(\frac{3}{2}\right)_{n-k}}, \frac{\zeta_{1}^{n}}{n !}=\sum_{k=0}^{n} \frac{(-1)^{k}(I+\mathscr{A})_{n}\left[(I+\mathscr{A})_{k}\right]^{-1} L_{k}^{\mathscr{A}}\left(\zeta_{1}\right)}{(n-k) !} .
$$


Using this in the series

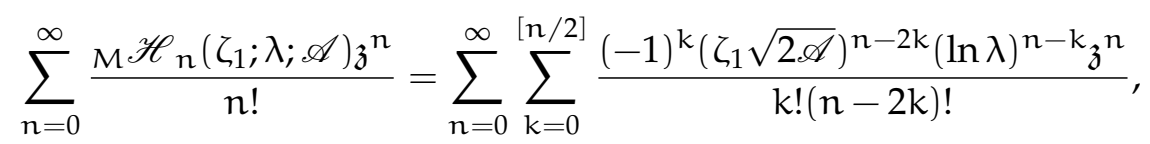

we get the other results.

\section{Integral representation}

Several integral involving modified Hermite matrix polynomials ${ }_{M} \mathscr{H}_{n}\left(\zeta_{1} ; \lambda ; \mathscr{A}\right)$ are as follows.

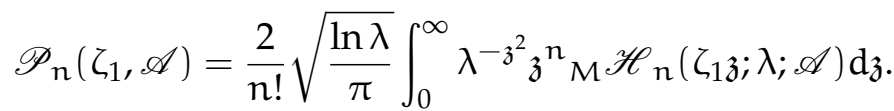

$$
\begin{aligned}
& \int_{-\infty}^{\infty} \lambda^{\frac{-\mathfrak{z}^{2} \mathscr{A}}{2}} \mathfrak{z}^{\mathrm{n}}{ }_{M} \mathscr{H}_{\mathrm{n}}\left(\zeta_{1} \mathfrak{z} ; \lambda ; \mathscr{A}\right) \mathrm{d} \mathfrak{z}=2 \mathrm{n} ! \sqrt{\frac{\pi}{\ln \lambda}} \sum_{\mathrm{k}=0}^{[\mathrm{n} / 2]} \frac{(-1)^{\mathrm{k}}\left(\zeta_{1} \sqrt{2 \mathscr{A}}\right)^{\mathrm{n}-2 \mathrm{k}}(2 / \mathscr{A})^{\mathrm{n}-\mathrm{k}+1 / 2}(1 / 2)_{\mathrm{n}-\mathrm{k}}}{\mathrm{k} !(\mathrm{n}-2 \mathrm{k}) !} . \\
& \mathrm{M} \mathscr{H}_{\mathrm{n}}\left(\zeta_{1} ; \lambda ; \mathscr{A}\right)=(2 \ln \lambda)^{\mathrm{n}+1} \lambda^{\zeta_{1}^{2}} \int_{\zeta_{1}}^{\infty} \lambda^{-\mathfrak{z}^{2}} \mathfrak{z}^{\mathrm{n}+1} \mathscr{P}_{\mathrm{n}}\left(\zeta_{1} / \mathfrak{z}, \mathscr{A}\right) \mathrm{d} \mathfrak{z} \\
& \int_{-\infty}^{\infty} \lambda^{\frac{-\zeta_{1}^{2} \mathscr{A}}{2}} \zeta_{1}^{\mathrm{n}} \mathrm{M} \mathscr{H}_{\mathrm{n}-2 \mathrm{k}}\left(\zeta_{1} ; \lambda ; \mathscr{A}\right) \mathrm{d} \zeta_{1}=\frac{(\sqrt{2 \mathscr{A}})^{-2 \mathrm{k}} \mathrm{n} !}{\mathrm{k} !(\ln \lambda)^{\mathrm{k}}} \sqrt{\frac{2 \pi \mathscr{A}^{-1}}{\ln \lambda}} .
\end{aligned}
$$

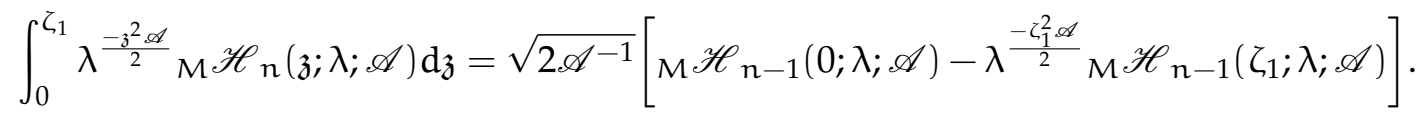

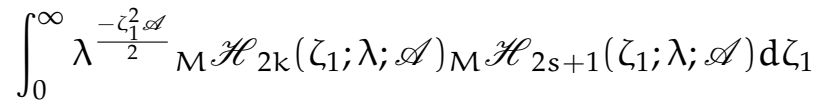

$$
\begin{aligned}
& =\frac{(-1)^{k+s} 2^{2(k+s)}\left(\frac{\mathscr{A}}{2}\right)^{-1 / 2}\left(\frac{1}{2}\right)_{k}\left(\frac{3}{2}\right)_{s}(\ln \lambda)^{k+s}}{1+2 s-2 k} .
\end{aligned}
$$

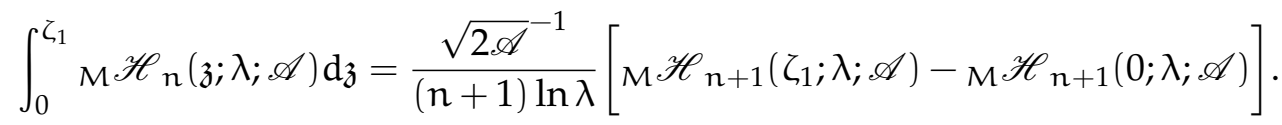

$$
\begin{aligned}
& \int_{-\infty}^{\infty} \lambda^{\frac{-\mathfrak{z}^{2} \mathscr{A}}{2}} \mathrm{M}^{\mathscr{H}_{2 n}}\left(\zeta_{1} \mathfrak{z} ; \lambda ; \mathscr{A}\right) \mathrm{d} \mathfrak{z}=\frac{(2 \mathrm{n}) ! \sqrt{\pi}\left(\frac{\mathscr{A}}{2}\right)^{-1 / 2}(\ln \lambda)^{\mathrm{n}-1 / 2}\left(\zeta_{1}^{2}-1\right)^{\mathrm{n}}}{\mathrm{n} !} .
\end{aligned}
$$

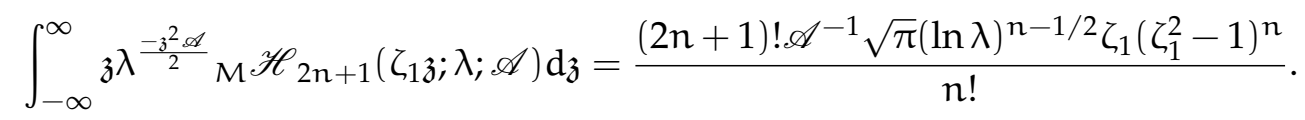

$$
\begin{aligned}
& \int_{-\infty}^{\infty} \mathfrak{z}^{\mathrm{n}} \lambda^{-\mathfrak{z}^{2}}{ }^{\mathrm{M}} \mathscr{H}_{\mathrm{n}}\left(\zeta_{1} ; \dot{\mathfrak{z}} ; \dot{A}\right) \mathrm{d} \mathfrak{z}=\frac{\mathrm{n} ! \sqrt{\pi}}{\sqrt{\ln \lambda}} \mathscr{P}_{\mathrm{n}}\left(\zeta_{1}, \mathscr{A}\right) \\
& \int_{-\infty}^{\infty} \lambda^{\frac{-\zeta_{1}^{2} \mathscr{A}}{2}} M^{\mathscr{H}_{2 n}}\left(\sqrt{2} \zeta_{1} ; \lambda ; \mathscr{A}\right) \mathrm{d} \zeta_{1}=\frac{(2 \mathrm{n}) ! \sqrt{\pi}\left(\frac{\mathscr{A}}{2}\right)^{-1 / 2}(\ln \lambda)^{\mathrm{n}-1 / 2}}{\mathrm{n} !} .
\end{aligned}
$$

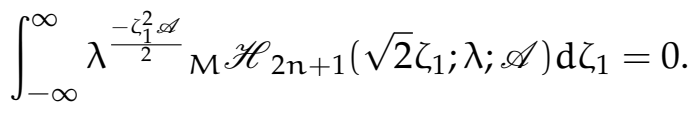

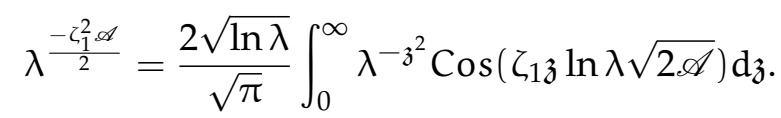

$$
\begin{aligned}
& \mathrm{M} \mathscr{H}_{2 \mathfrak{n}}\left(\zeta_{1} ; \lambda ; \mathscr{A}\right)=\frac{(-1)^{\mathrm{n}} 2^{2 \mathfrak{n}+1}(\ln \lambda)^{2 \mathfrak{n}+1 / 2}}{\sqrt{\pi}} \int_{0}^{\infty} \lambda^{-\mathfrak{z}^{2}} \mathfrak{z}^{2 \mathrm{n}} \operatorname{Cos}\left(\zeta_{1} \mathfrak{z} \ln \lambda \sqrt{2 \mathscr{A}}\right) \mathrm{d} \mathfrak{z} . \\
& \mathrm{M}^{\mathscr{H}_{2 \mathfrak{n}+1}}\left(\zeta_{1} ; \lambda ; \mathscr{A}\right)=\frac{(-1)^{\mathrm{n}} 2^{2 \mathfrak{n}+2}(\ln \lambda)^{2 \mathfrak{n}+1}}{\sqrt{\pi}} \int_{0}^{\infty} \lambda^{-\mathfrak{z}^{2}} \mathfrak{z}^{2 \mathfrak{n}+1} \operatorname{Sin}\left(\zeta_{1 \mathfrak{z}} \ln \lambda \sqrt{2 \mathscr{A}}\right) \mathrm{d} \mathfrak{z} .
\end{aligned}
$$




$$
\begin{aligned}
\Gamma(\mathrm{nI}+\mathscr{A}+\mathrm{I}) \int_{-1}^{+1}\left(1-\mathfrak{z}^{2}\right)^{\mathscr{A}-\mathrm{I} / 2}{ }_{\mathrm{M}} \mathscr{H}_{2 \mathrm{n}}\left(\sqrt{2 \mathscr{A}^{-1} \zeta_{1}} \mathfrak{z} ; \lambda ; \mathscr{A}\right) \mathrm{d} \mathfrak{z} \\
=(-1)^{\mathrm{n}} \sqrt{\pi}(2 \mathrm{n}) !(\ln \lambda)^{\mathrm{n}} \Gamma(\mathscr{A}+\mathrm{I} / 2) \mathrm{L}_{\mathrm{n}}^{(\mathscr{A}, \ln \lambda)}\left(\zeta_{1}\right) . \\
\Gamma(\mathrm{nI}+\mathscr{A}+\mathrm{I}) \int_{-\frac{1}{\sqrt{\ln \lambda}}}^{+\frac{1}{\sqrt{\ln \lambda}}}\left(1-\mathfrak{z}^{2} \ln \lambda\right)^{\mathscr{A}-\mathrm{I} / 2}{ }_{\mathrm{M}} \mathscr{H}_{2 \mathrm{n}}\left(\sqrt{2 \mathscr{A}^{-1} \zeta_{1}} ; \lambda ; \mathscr{A}\right) \mathrm{d} \mathfrak{z} \\
=(-1)^{\mathrm{n}} \sqrt{\pi}(2 \mathrm{n}) !(\ln \lambda)^{\mathrm{n}-1 / 2} \Gamma(\mathscr{A}+\mathrm{I} / 2) \mathrm{L}_{\mathrm{n}}^{(\mathscr{A})}\left(\zeta_{1}\right) .
\end{aligned}
$$

Where $\mathrm{L}_{n}^{(\mathscr{A})}\left(\zeta_{1}\right)$ is the Laguerre matrix polynomials see [21].

\section{Fractional integrals and derivatives of ${ }_{\mathrm{M}} \mathscr{H}_{\mathbf{n}}\left(\zeta_{1} ; \lambda ; \mathscr{A}\right)$}

By using the definitions of fractional integrals and fractional derivatives given in [32], we have obtained the following fractional integrals and fractional derivatives for modified Hermite matrix polynomials $\mathrm{M} \mathscr{H}_{\mathrm{n}}\left(\zeta_{1} ; \lambda ; \mathscr{A}\right)$ given as,

$$
\mathrm{I}^{\mu}\left\{{ }_{\mathrm{M}} \mathscr{H}_{\mathrm{n}}\left(\zeta_{1} ; \lambda ; \mathscr{A}\right)\right\}=\frac{\mathrm{M} \mathscr{H}_{\mathrm{n}+\mu}\left(\zeta_{1} ; \lambda ; \mathscr{A}\right)}{(1+\mathrm{n})_{\mu}(\sqrt{2 \mathscr{A}} \ln \lambda)^{\mu}}
$$

the Riemann-Liouville left side fractional integral:

$$
{ }_{\mathrm{a}} \mathrm{I}_{\zeta_{1}}^{\alpha}\left\{\mathrm{M} \mathscr{H}_{\mathrm{n}}\left(\zeta_{1}-\mathrm{a} ; \lambda ; \mathscr{A}\right)\right\}=\frac{\mathrm{M}^{\mathscr{H}} \mathrm{n}+\alpha\left(\zeta_{1}-\mathrm{a} ; \lambda ; \mathscr{A}\right)}{(1+\mathrm{n})_{\alpha}(\sqrt{2 \mathscr{A}} \ln \lambda)^{\alpha}}
$$

the Riemann-Liouville right side fractional integral:

$$
\zeta_{1} \mathrm{I}_{\mathrm{c}}^{\alpha}\left\{{ }_{M} \mathscr{H}_{\mathrm{n}}\left(\mathrm{c}-\zeta_{1} ; \lambda ; \mathscr{A}\right)\right\}=\frac{\mathrm{M} \mathscr{H}_{\mathrm{n}+\alpha}\left(\mathrm{c}-\zeta_{1} ; \lambda ; \mathscr{A}\right)}{(1+\mathrm{n})_{\alpha}(\sqrt{2 \mathscr{A}} \ln \lambda)^{\alpha}}
$$

the Weyl integral of ${ }_{\mathrm{M}} \mathscr{H}_{\mathrm{n}}\left(\zeta_{1} ; \lambda ; \mathscr{A}\right)$ of order $\alpha$ :

$$
\zeta_{1} W_{\infty}^{\alpha}\left\{{ }_{M} \mathscr{H}_{\mathrm{n}}\left(\zeta_{1} ; \lambda ; \mathscr{A}\right)\right\}=\frac{(-1)^{\alpha}{ }_{\mathrm{M}} \mathscr{H}_{\mathrm{n}+\alpha}\left(\zeta_{1} ; \lambda ; \mathscr{A}\right)}{(1+\mathrm{n})_{\alpha}(\sqrt{2 \mathscr{A}} \ln \lambda)^{\alpha}},
$$

the Erdelyi-Kober operator of first kind for ${ }_{M} \mathscr{H}_{\mathrm{n}}\left(\zeta_{1} ; \lambda ; \mathscr{A}\right)$ :

$$
\mathrm{I}\left[\alpha, \eta, \mathrm{M} \mathscr{H}_{\mathrm{n}}\left(\zeta_{1} ; \lambda ; \mathscr{A}\right)\right]=\frac{\left(\sqrt{2 \mathscr{A}} \zeta_{1} \ln \lambda\right)^{\mathrm{n}}}{(1+\mathrm{n}+\eta)_{\alpha}}{ }_{4} \mathrm{~F}_{2}\left[\begin{array}{c}
\Delta(2,-\mathrm{n}), \Delta(2,-\alpha-\mathrm{n}-\eta) ; \\
\Delta(2,-\mathrm{n}-\eta) ;
\end{array}\right.
$$

the Erdelyi-Kober operator of second kind for ${ }_{\mathrm{M}} \mathscr{H}_{\mathrm{n}}\left(\zeta_{1} ; \lambda ; \mathscr{A}\right)$ :

$$
\mathrm{I}\left[\alpha, \eta, \mathrm{M} \mathscr{H}_{\mathrm{n}}\left(\zeta_{1} ; \lambda ; \mathscr{A}\right)\right]=\frac{\left(\sqrt{2 \mathscr{A}} \zeta_{1} \ln \lambda\right)^{\mathrm{n}}}{(1-\mathrm{n}+\eta)_{\alpha}}{ }_{4} \mathrm{~F}_{2}\left[\begin{array}{cc}
\Delta(2,-\mathrm{n}+\eta), \Delta(2,-\mathrm{n}+\eta+1) ; & \frac{-2 \mathscr{A}^{-1}}{\zeta_{1}^{2} \ln \lambda} \\
\Delta(2,-n+\eta+\alpha) ;
\end{array}\right],
$$

the Saigo integral operator of first kind:

$$
\begin{aligned}
\mathrm{I}_{0+}^{\alpha, \beta, \eta}\left\{{ }_{M} \mathscr{H}_{\mathrm{n}}\left(\zeta_{1} ; \lambda ; \mathscr{A}\right)\right\}= & \frac{(\sqrt{2 \mathscr{A}} \ln \lambda)^{\mathrm{n}} \Gamma(1+\mathrm{n}) \Gamma(1+\mathrm{n}+\eta-\beta) \zeta_{1}^{\mathrm{n}-\beta}}{\Gamma(1+\mathrm{n}-\beta) \Gamma(1+\mathrm{n}+\eta+\alpha)} \\
& \times{ }_{4} \mathrm{~F}_{2}\left[\begin{array}{c}
\Delta(2,-\mathrm{n}+\beta), \Delta(2,-\mathrm{n}-\eta-\alpha) ; \\
\Delta(2,-\mathrm{n}-\eta+\beta) ;
\end{array}\right],
\end{aligned}
$$


the Saigo integral operator of second kind:

$$
\begin{aligned}
& \mathrm{I}_{0-}^{\alpha, \beta, \eta}\left\{{ }_{M} \mathscr{H}_{\mathrm{n}}\left(\zeta_{1} ; \lambda ; \mathscr{A}\right)\right\}=\frac{(\sqrt{2 \mathscr{A}} \ln \lambda)^{\mathrm{n}} \Gamma(1-\mathrm{n}) \Gamma(1-\mathrm{n}+\eta-\beta) \zeta_{1}^{\mathrm{n}-1}}{\Gamma(1-\mathrm{n}-\beta) \Gamma(1-\mathrm{n}+\eta+\alpha)}
\end{aligned}
$$

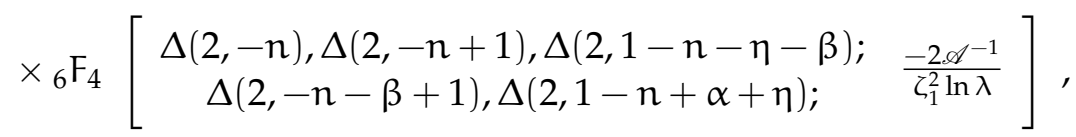

the left sided Riemann-Liouville fractional derivative of order $\alpha$ :

$$
{ }_{\mathrm{a}} \mathrm{D}_{\zeta_{1}}^{\alpha}\left\{\mathrm{M} \mathscr{H}_{\mathrm{n}}\left(\zeta_{1}-\mathrm{a} ; \lambda ; \mathscr{A}\right)\right\}=\frac{(\sqrt{2 \mathscr{A}} \ln \lambda)^{\alpha} \Gamma(1+\mathrm{n})}{\Gamma(1+\mathrm{n}-\alpha)} \mathrm{M} \mathscr{H}_{\mathrm{n}-\alpha}\left(\zeta_{1}-\mathrm{a} ; \lambda ; \mathscr{A}\right),
$$

the Right sided Riemann-Liouville fractional derivative of order $\alpha$ :

$$
\zeta_{1} D_{\mathrm{c}}^{\alpha}\left\{\mathrm{M} \mathscr{H}_{\mathrm{n}}\left(\mathrm{c}-\zeta_{1} ; \lambda ; \mathscr{A}\right)\right\}=\frac{(\sqrt{2 \mathscr{A}} \ln \lambda)^{\alpha} \Gamma(1+\mathrm{n})}{\Gamma(1+\mathrm{n}-\alpha)} \mathrm{M} \mathscr{H}_{\mathrm{n}-\alpha}\left(\mathrm{c}-\zeta_{1} ; \lambda ; \mathscr{A}\right),
$$

the Weyl fractional derivative of order $\alpha$ :

$$
{ }_{\zeta_{1}} \mathrm{D}_{\infty}^{\alpha}\left\{{ }_{\mathrm{M}} \mathscr{H}_{\mathrm{n}}\left(\zeta_{1} ; \lambda ; \mathscr{A}\right)\right\}=(-\mathrm{n})_{\alpha}(\sqrt{2 \mathscr{A}} \ln \lambda)^{\alpha}{ }_{\mathrm{M}} \mathscr{H}_{\mathrm{n}-\alpha}\left(\zeta_{1} ; \lambda ; \mathscr{A}\right)
$$

\section{Acknowledgment}

The author K. S. Nisar express his thanks to the Deanship of Scientific Research (DSR), Prince Sattam bin Abdulaziz University, Saudi Arabia, for providing facilities and support.

\section{References}

[1] R. S. Batahan, A new extension of Hermite matrix polynomials and its applications, Linear Algebra Appl., 419 (2006), 82-92. 1

[2] R. Bellman, Introduction to Matrix Analysis, McGraw-Hill Book Co., New York-Düsseldorf-London, (1970). 1

[3] F. Brafman, Some generating functions for Laguerre and Hermite polynomials, Canadian J. Math., 9 (1957), 180-187.

[4] E. Defez, L. Jódar, Some applications of the Hermite matrix polynomials series expansions, J. Comput. Appl. Math., 99 (1998), 105-117. 1, 1

[5] N. Dunford, J. T. Schwartz, Linear Operators, part I: General Theory, Interscience Publishers, New York, (1958).

[6] A. J. Durán, W. Van Assche, Orthogonal Matrix Polynomials and Higher Order Recurrence Relations, Linear Algebra Appl., 219 (1995), 261-280.

[7] L. Jódar, R. Company, Hermite Matrix Polynomials and Second Order Matrix Differential Equations, J. Approx. Theory Appl. (N.S.), 12 (1996), 20-30. 1, 2

[8] L. Jódar, R. Company, E. Navarro, Laguerre Matrix Polynomials and Systems of Second Order Differential Equations, Appl. Numer. Maths., 15 (1994), 53-63. 8

[9] L. Jódar, J. C. Cortés, On the hypergeometric matrix function, J. Comput. Appl. Math., 99 (1998), 205-217. 1, 1

[10] L. Jódar, J. C. Cortés, Closed form general solution of the hypergeometric matrix differential equation, Math. Comput. Modelling, 32 (2000), 1017-1028. 1

[11] L. Jódar, E. Defez, Some new matrix formulas related to Hermite matrix polynomials theory, International Workshop on Orthogonal Polynomials in Mathematical Physics (Univ. Carlos III Madrid, Leganés), 1996 (1996), 93-101. 1

[12] L. Jódar, E. Defez, A connection between Laguerre's and Hermite's matrix polynomials, Appl. Math. Lett., 11 (1998), 13-17. 1

[13] L. Jódar, E. Defez, On Hermite matrix polynomials and Hermite matrix function, J. Approx. Theory Appl. (N.S.), 14 (1998), 36-48. 1

[14] L. Jódar, E. Defez, E. Ponsoda, Matrix Quadrature Integration and Orthogonal Matrix Polynomials, Congr. Numer., 106 (1995), 141-153. 1

[15] W. A. Khan, Certain results for the Hermite and Chebyshev polynomials of 2-variables, Ann. Commun. Math., 2 (2019), 84-90. 1

[16] W. A. Khan, I. A. Khan, M. Ali, Degenerate Hermite poly-Bernoulli numbers and polynomials with q-parameter, Stud. Univ. Babes-Bolyai Math., 65 (2020), 3-15. 1 
[17] M. A. Khan, A. H. Khan, V. Singh, Study of Gegenbauer matrix Polynomials via matrix functions and their properties, Asian J. Math. Comput. Res., 16 (2017), 197-207. 8

[18] W. A. Khan, M. A. Pathan, On generalized Lagrange-Hermite-Bernoulli and related polynomials, Acta et Commentationes Universitatis Tartuensis de Mathematica, 23 (2019), 211-224. 1

[19] N. U. Khan, T. Usman, Certain generating functions of Hermite-Bernoulli-Legendre polynomials, Ufa Math. J., 10 (2018), 118-126.

[20] N. U. Khan, T. Usman, W. A. Khan, A new class of Laguerre-based generalized Hermite-Euler polynomials and its properties, Kragujevac J. Math., 44 (2020), 89-100. 1

[21] N. N. Lebedev, Special Functions and Their Applications, Dover Publications, New York, (1972). 9

[22] M. S. Metwally, M. T. Mohamed, A. Shehata, On Hermite-Hermite matrix polynomials, Math. Bohem., 133 (2008), 421-434. 1

[23] I. Najfeld, T. F. Havel, Derivaties of the Matrix Exponential and their Computation, Adv. Appl. Math., 16 (1995), 321-375. 1

[24] K. S. Nisar, W. A. Khan, Notes on q-Hermite based unified Apostol type polynomials, J. Interdiscip. Math., 22 (2019), 1185-1203. 1

[25] E. D. Rainville, Special functions, Macmillan Co., New York, (1960). 1

[26] K. A. M. Sayyed, M. S. Metwally, R. S. Batahan, On generalized Hermite matrix polynomial, Electron. J. Linear Algebra, 10 (2003), 272-279. 1

[27] K. A. M. Sayyed, M. S. Metwally, R. S. Batahan, Gegenbauer matrix polynomials and second order matrix differential equations, Divulg. Mat., 12 (2004), 101-115.

[28] A. Shehata, A new extension of Hermite-Hermite matrix polynomials and their properties, Thai J. Math., 10 (2012), 433-444.

[29] A. Shehata, R. Bhukya, Some properties of Hermite matrix polynomials, J. Int. Math. Virtual Inst., 5 (2015), 1-17. 1

[30] V. Singh, M. A. Khan, A. H. Khan, On a Multivariable Extension of Laguerre Matrix Ploynomials, Electron. J. Math. Anal. Appl., 6 (2018), 223-240. 8

[31] H. M. Srivastava, W. A. Khan, H. Haroon, Some expansions for a class of generalized Humbert Matrix polynomials, Rev. R. Acad. Cienc. Exactas Fís. Nat. Ser. A Mat. RACSAM, 113 (2019), 3619-3634. 1

[32] H. M. Srivastava, H. L. Manocha, A treatise on generating functions, John Wiley \& Sons, New York, (1984). 10

[33] L. M. Upadhyaya, A. Shehata, On Legendre matrix polynomials and its applications, Int. Trans. Math. Sci. Comput., 4 (2011), 291-310. 8 